\title{
IEEE 802.11n Aggregation Performance Study for the Multicast
}

\author{
Yousri Daldoul $^{1,2}$, Toufik Ahmed ${ }^{2}$, Djamal-Eddine Meddour ${ }^{1}$ \\ ${ }^{1}$ France Telecom - Orange Labs, France \\ ${ }^{2}$ LaBRI, University of Bordeaux 1, France \\ \{yousri.daldoul, djamal.meddour\}@ orange-ftgroup.com, tad@labri.fr
}

\begin{abstract}
One of the major features proposed in the IEEE 802.11n amendment is the use of frame aggregation. Thus, two types of frame aggregations are defined: MAC Service Data Unit Aggregation (A-MSDU) and MAC Protocol Data Unit Aggregation (A-MPDU). As frame aggregation is mainly defined to increase the efficiency of the MAC layer, this feature is very needed to support multimedia traffic such as High Definition TV (HDTV). The multicast transport is another way to increase the MAC layer efficiency by transmitting the same information only once to many receivers. However, the 802.11 standard does not define a reliable multicast and consequently the $802.11 \mathrm{n}$ is unable to provide a reliable transport for multicast streams. The 802.11aa remains the only draft to handle the unreliability issue of the multicast, but the proposed solution is defined only for the legacy 802.11 standard and does not consider the aggregation feature of the $802.11 n$. In this paper we design a new extension for the 802.11aa in order to take a full advantage of the 802.11n capabilities and we provide a new analytical model to evaluate the performance of the MAC layer. We particularly study the impact of the frame aggregation type on the MAC throughput for the multicast transport. Our results show that in an ideal channel (no losses), A-MSDU slightly outperforms A-MPDU when using the data rate of $6.5 \mathrm{Mbps}$ and both schemes have the same performance with rates of 130 and 300 Mbps. However the use of A-MPDU aggregation becomes more efficient in a noisy channel.
\end{abstract}

\section{INTRODUCTION}

The IEEE 802.11n [1] is known to be the next generation technology for the high throughput wireless networks. It allows data rates up to $600 \mathrm{Mbps}$. With its high capacity, this solution becomes commonly used within new communication devices.

The reduced efficiency of the legacy 802.11 MAC layer was firstly resolved by $802.11 \mathrm{e}$ using the Block frame and the Block Acknowledgement transfers, and then enhanced by 802.11n using frame aggregation. Thus two aggregation schemes are defined: MAC Service Data Unit Aggregation (AMSDU) and MAC Protocol Data Unit Aggregation (A-MPDU).

Although the data rate of the PHY layer has considerably increased within the 802.11 n compared to the legacy standard, the unreliability problem of the multicast remains unresolved. Like the legacy 802.11 , the 802.11n does not use any feedback policy when a frame is transmitted to a group address (broadcast or multicast). Hence the reliability of the multicast is hugely reduced compared to the unicast. In order to provide a reliable multicast transport, the 802.11aa [3] defines a new
Acknowledgement (ACK) policy for the 802.11. However this solution does not consider the frame aggregation feature of the 802.11n.

In this paper we design a new extension to the 802.11aa amendment in order to take a full advantage of the $802.11 \mathrm{n}$ capabilities. Then, we evaluate through an analytical model the performance of A-MSDU and A-MPDU aggregation schemes to transport a reliable multicast traffic. We particularly show how the encapsulated MSDUs' size and the bit error rate affect the efficiency of the MAC layer.

The remainder of this paper is organized as follows. Section II introduces related work of some proposed multicast protocols we have studied, discussing their compatibility with $802.11 \mathrm{n}$ and highlighting their problems and drawbacks. Changes brought to the 802.11 aa are described in section III. In section IV we provide our analytical model. We devote section $\mathrm{V}$ to show the performance of the two aggregation schemes. Finally, in section VI, we conclude.

\section{RELATED WORKS}

\section{II-A. State of the art of reliable multicast proposals}

Many protocols were designed to address the unreliability of the multicast in the 802.11 WLAN [3-7]. They can be classified into two categories: ACK-based [3,4,5] and Negative Acknowledgement (NAK) based [6,7] protocols. The ACKbased protocols use a similar concept to that of the unicast ACK and they require each multicast member to send an explicit ACK. Most of them can migrate easily to $802.11 \mathrm{n}$ since the sender performs the MAC layer recovery based on a trustworthy reply. However their performance should be studied again when used with the new aggregation feature.

In the NAK-based protocols, the receiver will reply with a NAK only if the frame is received with errors. In some proposals, the implementation of the protocol is joined with the selection of a leader for the multicast group, where the leader is the only responder with an ACK in case of reception success. The implementation of a NAK based protocol remains a challenging task as it requires resolving two constraints. 1) Eliminating cases where the leader's ACK signal strength may hide a multicast member's NAK, 2) Deciding whether or not a NAK should be sent, since the received information within a

\footnotetext{
978-1-4577-2028-4/11/\$26.00 @2011 IEEE
} 
faulty frame is not coherent and is not enough to build the appropriate feedback in a reliable way. The former constraint may be resolved by selecting a leader on the basis of the lowest link quality criterion [7]. The latter is resolved by using additional control frames (RTS/CTS, CTS-To-Self) to carry the trustworthy information which will be used to build a feedback. Most of NAK-based protocols are inappropriate for the AMPDU aggregation scheme since a NAK is used to acknowledge only one MPDU.

\section{II-B. Aggregation performance studies}

In the literature, many works have studied the aggregation performance of 802.11n [8-10]. In [8] authors evaluated the AMSDU, the A-MPDU and the two-level aggregation schemes, i.e. when one or more of the aggregated MPDUs encapsulate an A-MSDU. They proved that the two-level scheme is the most efficient one. In [9], authors proposed a novel analytic model to compare A-MSDU and A-MPDU schemes with UDP and TCP. However they limited their evaluation of the AMSDU scheme to the maximum supported length of a single A-MSDU without considering the two-level scheme. The second weakness of the model is the use of an infinite retry limit which is not a realistic value in a real network. In [10], authors focused on the overhead's impact on the used scheme. The major weakness of the paper is ignoring the overhead generated by channel contention and feedbacks.

All mentioned studies have investigated only the unicast transport. To our knowledge, the multicast mode has not been thoroughly studied, and the few papers which have studied it, have mainly focused on increasing its reliability. Therefore, analyzing the impact of the aggregation scheme on the MAC efficiency has been ignored for the multicast transport. In this paper we fill this gap and we introduce our analytical model.

To achieve our evaluation of the MAC aggregation schemes, we need a reliable multicast transport allowing the source to retransmit when a reception error occurs. However, no ACK policy was defined for the multicast in the 802.11n. Hence we define a new extension for 802.11aa compliant with the $802.11 \mathrm{n}$ aggregation schemes. We use this extension to develop our analytical model.

\section{OVERVIEW OF THE 802.11N AND 802.11AA AMENDMENTS}

\section{III-A. Presentation of the 802.11 n amendment}

The 802.11n is designed to define the new version of High Throughput (HT) Wireless Local Area Networks (WLAN). Its theoretical PHY data rate may reach 600Mbps. Hence, many features were defined to enhance the performance of this amendment compared to the 802.11 standard. In this paper we focus on the frame aggregation feature only and we present both of the aggregation schemes: A-MSDU and A-MPDU.

The A-MSDU scheme allows the aggregation of several MSDUs into the same MPDU. The destination address (DA) and sender address (SA) of each aggregated MSDU must match with the receiver address (RA) and transmitter address (TA) of the MPDU MAC header. All MSDUs within an A-
MSDU should belong to the same traffic identifier (TID). The maximum length of an A-MSDU is 7935 bytes. The A-MSDU aggregation reduces the transmission overhead since only one MAC header is generated for all the aggregated MSDUs. However it becomes inefficient in noisy channels: if only one MSDU is corrupted, the entire A-MSDU is rejected and all the aggregated MSDUs are lost.

The A-MPDU scheme allows the aggregation of several MPDUs into the same PHY layer frame (PPDU). The maximum supported length for an A-MPDU is 65535 bytes. In an A-MPDU each MPDU is encapsulated with its MAC header and CRC. Hence the corruption of an MPDU is separately detected and does not affect the entire A-MPDU. However, the A-MPDU aggregation requires more overhead than A-MSDU which becomes heavy for small MSDUs.

In order to provide a reliable unicast transport, both 802.11 and 802.11n define an Acknowledgement (ACK) policy. Moreover, 802.11n allows the use of the immediate Block ACK (BACK) policy with single PPDUs carrying an A-MPDU when the ACK policy of the A-MPDU is set to "Implicit Block ACK" (i.e. the ACK policy subfield of at least one aggregated MPDU is set to "Normal ACK"). The legacy ACK policy is used with frames encapsulating a single MPDU (i.e. MSDU or A-MSDU) and requiring an immediate feedback.

Unlike the unicast, the 802.11n multicast transport does not use any feedback policy. Besides, the 802.11 n forbids non-Access Point (AP) stations (STAs) from transmitting group addressed frames and only the AP is allowed to transmit such frames.

\section{III-B. Presentation of the 802.11aa amendment}

The 802.11aa amendment [3] defines the More Reliable Groupcast (MRG) service in order to support a robust and reliable multicast audio/video streaming over the 802.11 WLAN. This service provides three ACK policies: MRGUnsolicited-Retry, MRG-Directed Multicast Service (DMS) and MRG-Block-Ack.

When using the MRG-Unsolicited-Retry ACK policy, the sender defines a retry limit, say " $N$ ", and then transmits each multicast frame " $N$ " times without waiting for any feedback after each transmission. This policy generates a significant overhead particularly when the value of " $N$ " is high, and becomes useless when the channel quality is good.

The MRG-DMS sends each multicast frame in unicast mode to each multicast group member. Therefore, this ACK policy guarantees the same unicast reliability level to multicast traffic at the cost of the bandwidth. DMS can be used to stream a standard multicast traffic to a few receivers' number. However it does not scale well for high throughput traffic like High Definition (HD) TV streams.

The MRG-Block-Ack feedback policy is designed to operate with the block frame transfer. For such transfer, the receiver is allowed to send a BACK only when it receives an explicit Block ACK Request (BAR) frame. The MRG-Block-Ack feedback procedure is illustrated in Fig. 1. 


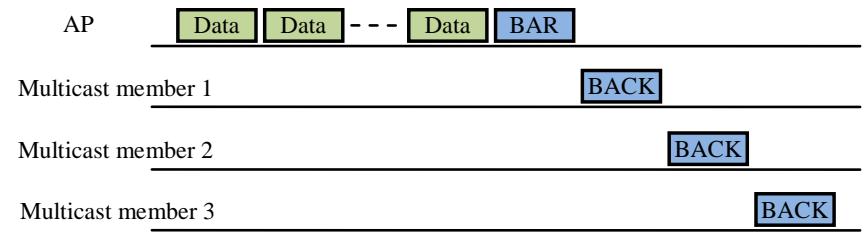

Figure 1. Typical frame exchange scenario with MRG-Block-Ack policy

Since MRG service is defined for multicast transmissions, the MRG BAR is slightly changed compared to that of the unicast in order to add the following information: the multicast members and the acknowledgement order. When a BAR is used as part of MRG-Block-Ack, a new field with a variable length called "MRG BAR information field" is added to the BAR. This field allows the AP to ask for a feedback from up to 2008 associated STAs. Thus, the field's length may reach 253 bytes. This length may introduce a useless overhead since multicast groups in a WLAN have limited members typically fewer than 8 , and becomes annoying when each multicast frame needs to be individually acknowledged.

Although MRG-Block-Ack provides the best reliability to bandwidth ratio among the three proposed ACK policies, it does not allow an optimized utilization of the 802.11n frame aggregation feature. Since an A-MPDU has a significant supported length allowing the aggregation of many MPDUs into the same PPDU, it becomes more convenient to request a feedback per frame particularly for real-time traffic subject to low latency constraints.

Concerning the multicast group initialization, the 802.11aa amendment does not define a particular procedure, but proposes the use of IGMP [11] snooping to achieve group membership detection.

\section{The MRG-IMPLICIT-BLOCK-ACK FEEDBACK POLICY}

In this section we introduce a new extension to the 802.11aa called the MRG-Implicit-Block-Ack (IBA) feedback policy. As for A-MPDUs transmitted in unicast with implicit ACK, the MRG-IBA is used to allow each A-MPDU to be acknowledged individually and immediately. In order to allow MRG-IBA to operate properly, 2 functions are required: membership management and ACK notification.

The membership management function allows the AP to attribute a Membership Identifier (MID) individually to each new detected member. In this paper we do not define the frame format used to notify the MID attribution for space constraints. The AP maintains a table called Group Membership Table (GMT) per multicast session. This table contains all the attributed MIDs for a specific session. An example of the GMT table with 4 members is illustrated in Table 1.

Table 1. GMT of a multicast session with address 01:00:5E:00:00:01

\begin{tabular}{|c|c|}
\hline Member address & MID \\
\hline 00:20:4E:38:A1:9B & 1 \\
00:20:7F:74:4E:25 & 2 \\
00:20:92:27:8A:75 & 4 \\
00:20:A6:61:1F:2B & 6 \\
\hline
\end{tabular}

When a multicast group contains 16 members or less, the highest attributed MID value should not exceed 16. Gaps may exist between attributed MIBs, and this may occur when a MIB is recovered after a member departure. If the members' number is higher than 16, all MIDs between 1 and 16 should be attributed. Thus, the AP performs MID reassignment when a MID between 1 and 16 becomes free. The choice of the value of 16 will be motivated in the remainder of this section.

The ACK notification function is implemented to assign an order for the feedbacks after each frame. In order to allow this function to operate properly we define a new-two-bytes-inlength field in the frame called the "MRG-IBA Bitmap field". This field is present in the MPDU header of a group addressed frame when both of the following conditions are satisfied: the MRG-IBA policy is used and the ACK policy of the frame is set to "Normal ACK". The new field is inserted between the HT control field and the data field of the MPDU.

Each bit of the MRG-IBA Bitmap field corresponds to a MID. Therefore, the AP requests that the member with a MID equal to " $N$ " to respond if the bit number " $N$ " is set to 1 and to forbear otherwise. As the bitmap field is 16 bits in length, up to 16 members may reply. We believe that a typical multicast group size in a WLAN does not exceed 16 members. However, when the size is higher than 16, the AP may choose between allowing only the first 16 members to reply till some participants leave the group, and switching to the MRG-BlockAck policy.

When the MRG-IBA agreement is established, multicast PPDUs encapsulating a single MPDU (i.e MSDU or A-MSDU) are also acknowledged using the MRG-IBA procedure. However, requested members reply with ACK frames instead of BACKs.

\section{MODEL DESCRIPTION}

In this section we evaluate the $802.11 \mathrm{n}$ aggregation schemes in the infrastructure mode. We build a Basic Service Set (BSS) of one AP and 8 associated STAs. All nodes are 802.11n compliant STAs. The AP delivers only one stream belonging to one multicast session and no traffic other than the multicast one is transmitted on the medium. All high level packets are delivered using UDP. The MRG-IBA agreement is established at the beginning of the session.

We define a retry limit of 7 for each MPDU and we suppose that MPDUs' failures within an A-MPDU are not correlated. All encapsulated MSDUs have 1500 bytes' length $(L)$. Hence an A-MPDU can aggregate at most 42 MPDUs encapsulating a single MSDU, and an A-MSDU can carry no more than 5 MSDUs. To evaluate the A-MSDU scheme with more than 5 aggregated MSDUs, we use the two-level aggregation scheme where several A-MSDUs are aggregated within an A-MPDU.

We assume that the PHY frame header is always received correctly since it has a small size compared to the data part of the frame and is always transmitted at the lowest but the most robust available data rate. All feedbacks (BACK and ACK 
frames) are transmitted at the same data rate used to deliver the multicast frame. We also assume that an ACK/BACK frame is always received correctly given that it has a small size compared to that of the data frame. Moreover, we consider that all PPDUs are transmitted during transmission opportunities (TXOP) which are contention free periods. Hence, the backoff procedure is not performed before a frame transmission.

Results are obtained for the following 2 mandatory data rates: 6.5 and $130 \mathrm{Mbps}$, and for the optional data rate of $300 \mathrm{Mbps}$. We consider that only the HT-greenfield format is used for all transmitted PPDUs.

\section{$V$-A. Mathematical tools:}

Let $p(i)$ be the bit error rate for Member " $i$ " $\left(M_{i}\right)$, for $i=1 . . K$, where $K$ is the multicast group size. We define $X$ as the number of transmission attempts. The probability for a given member $M_{i}$ to receive correctly a packet in any of the $N$ first transmissions is given in Equation (1):

$P^{i}(X \leq N)=1-(1-P s(i))^{N}$

Then we derive the probability to serve all the $K$ receivers in any of the $N$ first transmissions in Equation (2):

$P(X \leq N)=\prod_{i=1}^{K}\left(1-(1-P s(i))^{N}\right)$

where $P s(i)$ is the packet success rate for $M_{i}$. Therefore, $P s(i)$ is a function of the data length $n$ and $p(i)$, and is illustrated in Equation (3):

$\operatorname{Ps}(\mathrm{i})=(1-\mathrm{p}(\mathrm{i}))^{\mathrm{n}}$

It is obvious that the probability to receive correctly a packet in $X=0$ attempts is null, hence $P(X=0)=P^{i}(X=0)=0$. We also express the MAC layer efficiency as follows:

$E=($ data_length/PHY_rate $) /$ transmission_time

As the MAC efficiency in the multicast transmission mode may depend on the receiver's channel quality, we evaluate the AP MAC efficiency as perceived by each multicast member. Hence, this efficiency as perceived by a given member $M_{i}$ is illustrated in Equation (5):

$E^{i}=\left(\right.$ total_received_data $\left./ P H Y \_r a t e\right) /$ transmission_time

\section{$V$-B. A-MPDU aggregation performance:}

As we assumed that the PHY frame header is always received correctly, MPDU losses within an A-MPDU are independent from each other. Consequently we calculate the overhead per aggregated MPDU as the sum of the MPDU's overhead and the A-MPDU's transmission overhead divided by the number of aggregated MPDUs. This assumption is valid since we are transmitting MPDUs of the same length. We obtain the average A-MPDU transmission time in Equation (6) for a number of $A_{M P D U s}$ aggregated MPDUs and a retry limit of 7:

$$
\begin{gathered}
T_{A-M P D U}=A_{M P D U S} \cdot \sum_{N=1}^{7}\left(\frac{T_{\text {transmission _over head }}}{A_{M P D U S}}+T_{M P D U}\right) \\
.(1-P(X \leq N-1))
\end{gathered}
$$

$T_{\text {transmission_overhead }}=S I F S+T_{P H Y \_ \text {header }}$

$$
+K\left(S I F S+T_{B A C K}\right)
$$

and:

$T_{M P D U}=\left(M P D U \_\right.$overhead $\left.+L\right) / R$

We define, in Equation (9), the mean received MPDUs for a given member $M_{i}, A_{M P D U s}^{i}$, in order to evaluate the AP MAC efficiency as perceived by $M_{i}$.

$A_{M P D U S}^{i}=A_{M P D U S} \cdot P^{i}(X \leq 7)$

Hence, we derive in Equation (10), the efficiency equation for the A-MPDU scheme as perceived by $M_{i}$ :

$E_{A-M P D U}^{i}=\frac{A_{M P D U S}^{i} \cdot L / R}{T_{A-M P D U}}$

V-C. A-MSDU aggregation performance:

We consider the two-level aggregation scheme in order to evaluate the MAC efficiency using the A-MSDU scheme when the length of aggregated MSDUs exceeds the maximum supported length of 7935 bytes of the A-MSDU. Thus, we consider two cases: $A_{M S D U S} \leq 5$ and $A_{M S D U S}>5$, where $A_{M S D U S}$ is the number of aggregated MSDUs. In the first case only one AMSDU, is transmitted. When $A_{M S D U s}>5$, the A-MPDU aggregation scheme is used, and each MPDU encapsulates an A-MSDU. Therefore the maximum supported length of an AMSDU is limited to 3839 bytes and no more than 2 MSDUs can be aggregated within an A-MSDU. For calculation simplicity, for $A_{M S D U s}>5$, we consider only even values of $A_{M S D U s}$ in order to obtain MPDUs with equal sizes.

Case 1: $A_{M S D U s} \leq 5$

In the A-MSDU scheme, if an aggregated MSDU is received with errors, the entire A-MSDU is rejected. Besides when the PHY frame carries only one A-MSDU, receivers will reply with ACK frames instead of BACKs. We derive the average A-MSDU transmission time in Equation (11) for $A_{M S D U s}$ aggregated MSDUs and a retry limit of 7:

$$
\begin{gathered}
T_{A-M S D U}=\sum_{N=1}^{7}\left(T_{\text {transmission _overhead }}+A_{M S D U S} . T_{M S D U}\right) \\
\cdot(1-P(X \leq N-1))
\end{gathered}
$$

where:

$$
\begin{gathered}
T_{\text {transmission_overhead }}=S I F S+T_{P H Y \_ \text {header }} \\
+T_{M P D U \_o v e r \text { head }}+K\left(S I F S+T_{A C K}\right)
\end{gathered}
$$

and:

$T_{M S D U}=\left(M S D U \_o v e r h e a d+L\right) / R$

The mean received MSDUs for a given member $M_{i}, A_{M S D U s}^{i}$, is expressed in Equation (14). This equation is similar to Equation (9), however the value of $P(X=N), N=1 . .7$, will be affected by the increased size of the MPDU.

$A_{M S D U S}^{i}=A_{M S D U s} \cdot P^{i}(X \leq 7)$

where: 
In Equation (15), we derive the efficiency equation for the AMSDU scheme as perceived by $M_{i}$ :

$E_{A-M S D U}^{i}=\frac{A_{M S D U S}^{i} \cdot L / R}{T_{A-M S D U}}$

\section{$\underline{\text { Case 2: } A_{M S D U s}>5}$}

A-MSDU evaluation for $A_{M S D U s}>5$, follows equations defined for the A-MPDU scheme with few changes. Thus the average A-MPDU transmission time is given in Equation (16):

$$
\begin{gathered}
T_{A-M S D U}=\frac{A_{M S D U S}}{2} \cdot \sum_{N=1}^{7}\left(\frac{T_{\text {transmission_over head }}}{A_{M S D U S} / 2}+T_{M P D U}\right) \\
\cdot(1-P(X \leq N-1))
\end{gathered}
$$

where:

$T_{M P D U}=\left(T_{M P D U_{-} \text {overhead }}+2 . T_{M S D U_{-} \text {over head }}+2 . L\right) / R$

The expression of $T_{\text {transmission_overhead }}$ remains unchanged like in Equation (7). The values of $A_{M S D U s}^{i}$ and $E_{A-M S D U}^{i}$ follow Equation (14) and Equation (15) respectively.

\section{$V-D$. Analytical results:}

In Fig. 2 we evaluate the MAC layer efficiency of the AP for different sizes of A-MSDU and A-MPDU with three data rates: 6.5, 130 and 300Mbps. In Fig. 2 (a), (b) and (c) we consider an ideal channel case (i.e. bit error rate p=0). Except in Fig. 2 (a) where A-MSDU slightly outperforms A-MPDU, both aggregation schemes have almost the same efficiency. This is explained by the fact that the overhead difference between the two schemes has no effect when using high data rates. In a real channel however, the A-MPDU scheme has the highest performance since it carries MPDUs with smaller sizes than those carried using the A-MSDU scheme. In Fig. 2 (g), (h) and (i), when the bit error rate increases, increasing the size of an MPDU increases the packet error rate and hence increases the average transmission time. Thus using A-MSDU with up to 5 MSDUs does not provide a real enhancement to the MAC efficiency compared to the legacy mode (i.e. one MSDU per frame).

In Fig. 3 we show the evolution of the MAC efficiency when varying the bit error rate. As depicted in Fig. 3 (a), when no aggregation is used (only one MSDU per frame), the MAC layer has a reduced efficiency when using high data rates (130 and $300 \mathrm{Mbps}$ ). This is explained by the fact that the required time for transmitting the frame overhead and receivers' feedbacks is important compared to that required to deliver the encapsulated data. Fig. 3 (b) shows that an A-MPDU behaves better than an A-MSDU with 5 aggregated MSDUs in a bad channel condition. As depicted in Fig. 3 (d), the use of the aggregation feature improves the MAC efficiency with high data rates.
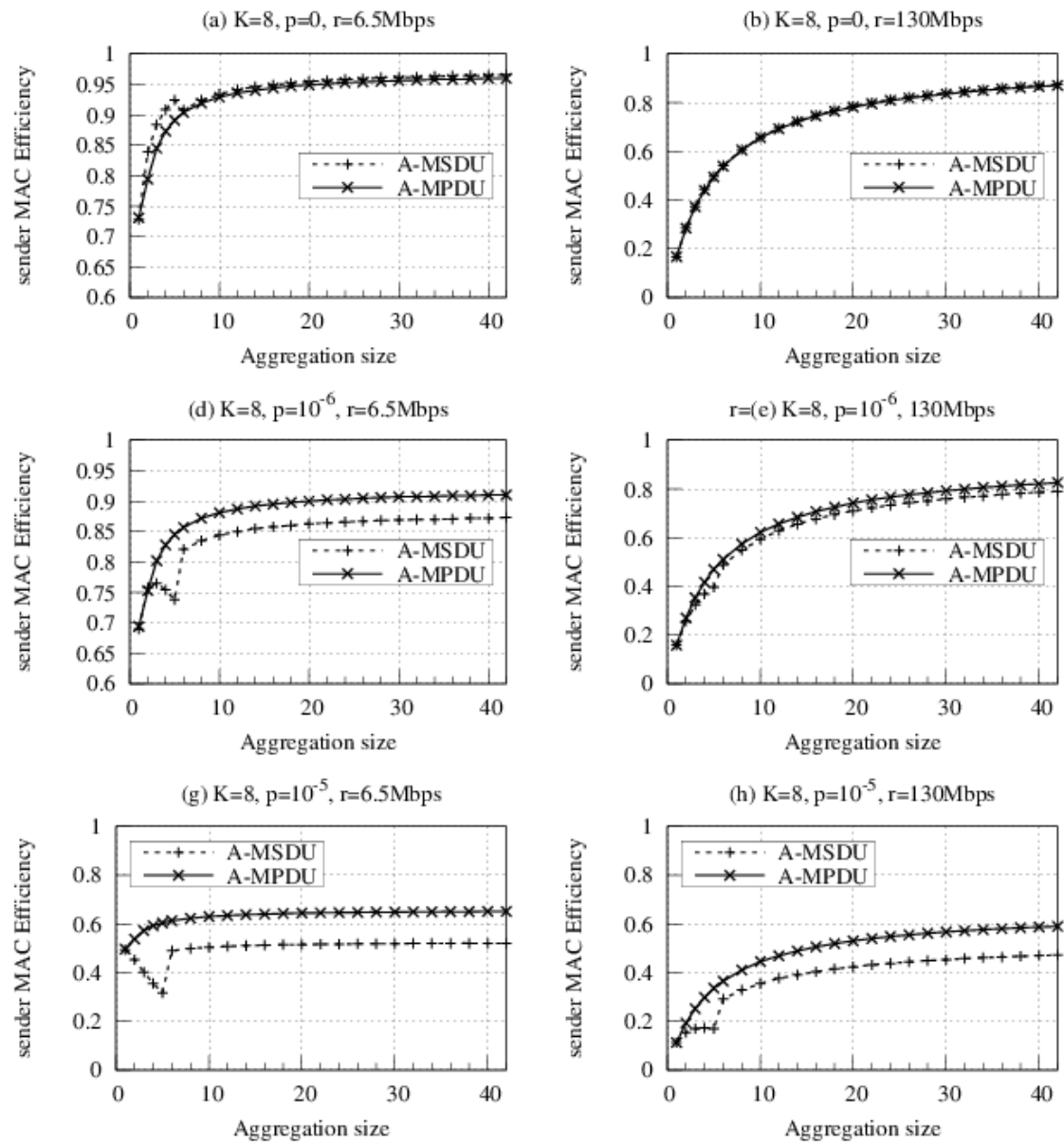
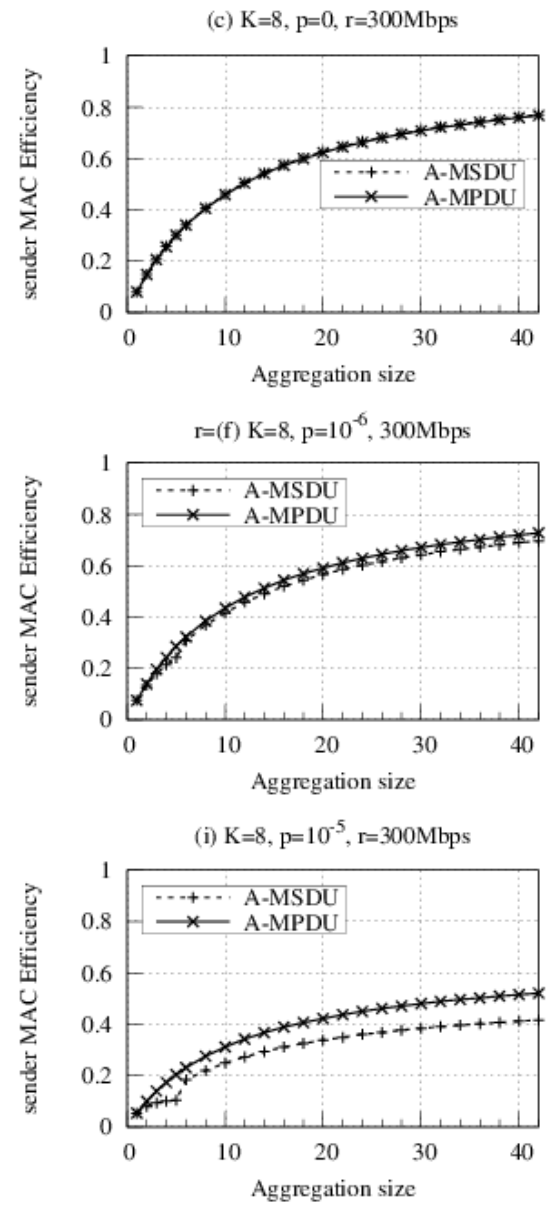

Figure 2. The AP MAC layer efficiency vs. the number of aggregated frames for a multicast group of 8 members 

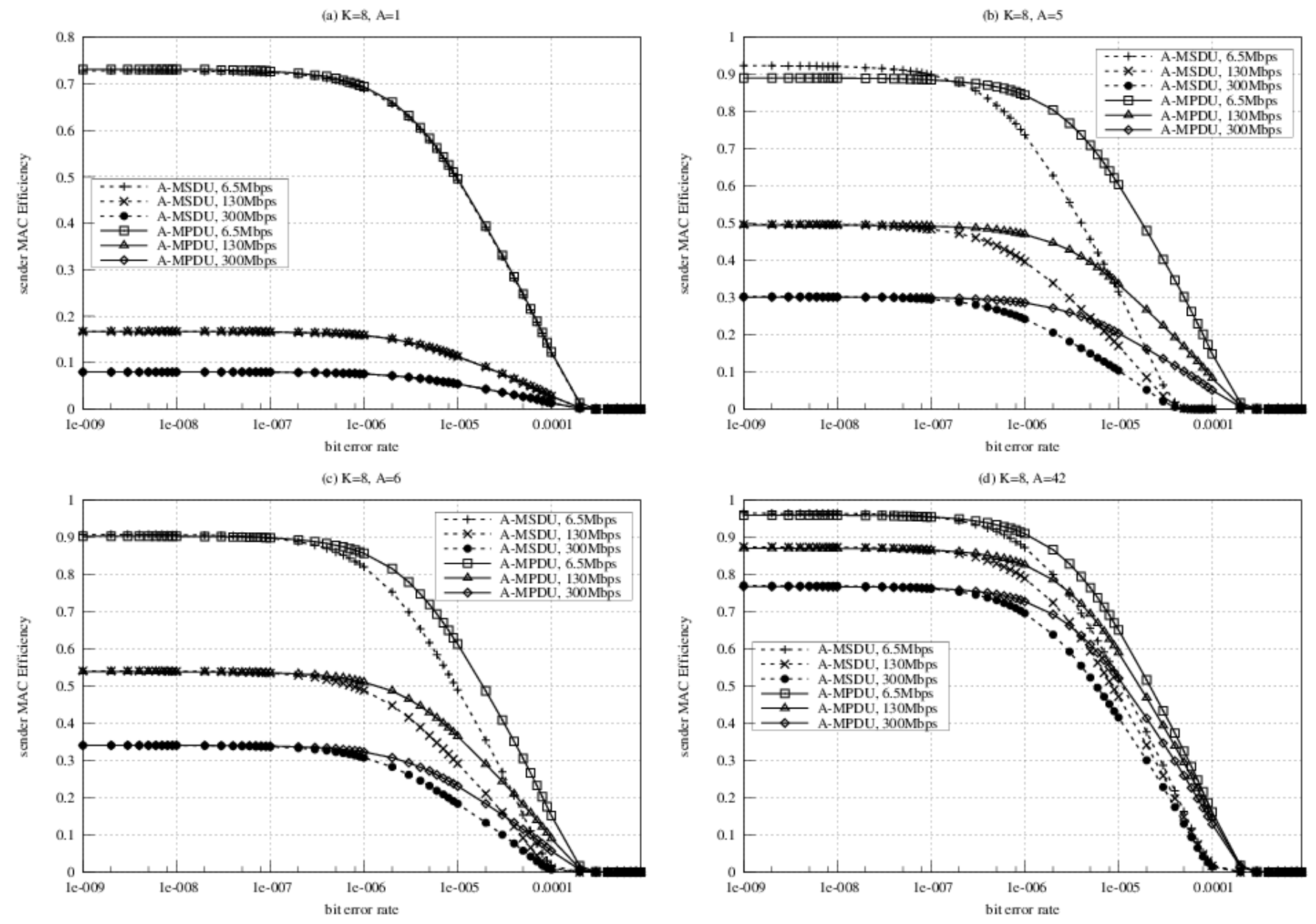

Figure 3. The AP MAC layer efficiency vs. the channel bit error rate for a multicast group of 8 members

\section{CONCLUSION}

In this paper we introduced the unreliability problem of the multicast within the IEEE 802.11 standard and we mainly focused on the 802.11aa solution and its extensions. We also presented both of the aggregation schemes proposed by the 802.11n amendment, namely A-MSDU and A-MPDU. Then we highlighted the weakness of 802.11aa extensions to provide a reliable multicast delivery of aggregated frames.

In order to enhance the 802.11aa amendment, we presented a new extension for the MRG service called MRG-IBA policy. This extension allows the amendment to take a full advantage of the 802.11n aggregation feature. The second contribution of this paper is defining a new analytical model to evaluate the performance of A-MSDU and A-MPDU aggregation schemes. This model is defined for a reliable multicast transport using the MRG-IBA recovery policy and allows the estimation of the MAC layer efficiency. Our model shows that both aggregation schemes have almost the same performance. However, A-MPDU behaves better than A-MSDU in a bad channel condition. In this study we limited our evaluation on the use of analytical results. Nevertheless, validating our model through simulations remains an important task which is missing in this paper but will be scheduled in our future works.

\section{REFERENCES}

[1] "Wireless LAN Medium Access Control (MAC) and Physical Layer (PHY) Specifications", IEEE std 802.11, 2007.

[2] "Wireless LAN Medium Access Control (MAC) and Physical Layer (PHY) specifications: Enhancements for Higher Throughput," IEEE std 802.11n, 2009.

[3] "Wireless LAN Medium Access Control (MAC) and Physical Layer (PHY) specifications: MAC Enhancements for Robust Audio Video Streaming," IEEE P802.11aa/D1.01, June 2010.

[4] Xiaoli Wang, Lan Wang, "Reliable Multicast Mechanism in WLAN with Extended Implicit MAC Acknowledgment", IEEE VTC, 2008.

[5] N. Choi, Y. Seok, T. Kwon, Y. Choi, "Transparent Unicast Translation to Improve Quality of Multicast over Wireless LAN,“IEEE CCNC, 2010.

[6] N. Choi, Y. Seok, T. Kwon, Y. Choi, T. Kwon. "Multicasting multimedia streams in IEEE 802.11 networks: a focus on reliability and rate adaptation," Springer Science+Business Media, LLC 2010.

[7] J. Miroll, Z. Li and Th. Herfet, "Wireless Feedback Cancellation for Leader-Based MAC Layer Multicast Protocols," IEEE ICSE, 2010.

[8] D. Skordoulis, Q. Ni, H. Chen, A. Stephens, C. Liu, and A. Jamalipour, "IEEE 802.11n MAC frame aggregation mechanisms for nextgeneration high-throughput WLANs," IEEE Wireless Communications, 2008.

[9] B. Ginzburg and A. Kesselman, "Performance analysis of A-MSDU and A-MPDU aggregation in IEEE 802.11n," IEEE SARNOFF, 2007.

[10] A. Saif, M. Othman, S. Subramaniam and N. AbdulHamid, "Impact of aggregation headers on aggregating small MSDUs in 802.11n WLANs," ICCAIE, 2010.

[11] B. Cain et al., "Internet Group Management Protocol, Version 3," RFC 3376, 2002. 